\section{Mandatory vaccination}

In the SARS commission report, Spring of fear, Justice Campbell stresses the importance of the precautionary principle and that "we cannot wait for scientific certainty before we take reasonable steps to reduce risk"1 In their CMAJ article, Gardam and Lemieux ${ }^{2}$ questioned the merit of mandatory influenza vaccination policies for health care providers because of relatively poor vaccine efficacy (approximately 60\%) and what they consider inadequate evidence.

Influenza vaccine is safe and provides net health benefits to both health care workers and their patients. Although we all want better vaccines, we use what is available. The 7-valent pneumococcal vaccine was just over $60 \%$ effective when introduced and the quadrivalent human papilloma virus vaccine in use protects against about two-thirds of cervical cancers.

Influenza vaccines are improving; the adjuvanted monovalent pandemic vaccine was over $90 \%$ effective. ${ }^{3}$ Flu vaccination reduced the risk of flurelated hospital admissions by $76.8 \%$ in study participants 50 years of age and older during the 2011-2012 season. ${ }^{4}$ Gardam and Lemieux ${ }^{2}$ do not clarify the acceptable level of effectiveness or how we decide this before the arrival of the virus and clarification of the virusvaccine match. Influenza vaccine is clearly "good enough" to save the lives of many patients. The threshold for not acting in the face of good evidence supporting patient safety should be high.

In their review of the ethical considerations, Ottenberg and colleagues ${ }^{5}$ found overwhelming scientific, ethical and legal justifications supporting mandating health care worker vaccination. They emphasized the professional obligations to benefit individual patients, to do no harm and also to protect public health in the face of preventable infectious disease.

Gardam and Lemieux ${ }^{2}$ suggest that mandatory vaccination may be legally challenged with implications for other mandatory programs. No evidence is provided to support this and mandatory vaccination is increasingly widespread in the United States, where institutions defending challenges on the basis of patient safety have won their cases.

If mandatory vaccination is a step too far for some of our colleagues, the British Columbia model is a wellrehearsed alternative. Staff unwilling or unable to be vaccinated can opt to wear a mask all winter. A large number of facilities using this model in the US have achieved coverage rates of $95 \%$.

Paul G. Van Buynder MBBS MPH, Elizabeth Bryce MD, Bonnie Henry MD MPH, Perry R. Kendall MBBS MSc Chief Medical Health Officer, Fraser Health Authority and Adjunct Professor Simon Fraser University (Van Buynder), Burnaby, BC; Regional Medical Director Infection Prevention and Control (Bryce), Vancouver Coastal Health Authority; BC Centre for Disease Control (Henry), University of British Columbia; and Provincial Health Officer, BC Ministry of Health and Clinical Professor, Faculty of Medicine, University of British Columbia (Kendall), Vancouver, BC

\section{References}

1. Campbell A. Spring of fear: The SARS Commission final report. Toronto $(\mathrm{ON})$ : SARS Commission; 2006. Available: www.ontla.on.ca/library/repository /mon/16000/268478.pdf (accessed 2013 Apr. 8).

2. Gardam M, Lemieux C. Mandatory influenza vaccination? First we need a better vaccine. CMAJ 2013;185:639-40.

3. Skowronski DM, Janjua NZ, De Serres G, et al. Effectiveness of AS03 adjuvanted pandemic $\mathrm{H} 1 \mathrm{~N} 1$ vaccine: case-control evaluation based on sentinel surveillance system in Canada, autumn 2009. BMJ 2011;342c:7297.

4. Talbot HK, Zhu Y, Chen Q, et al. Effectiveness of influenza vaccine for preventing laboratoryconfirmed influenza hospitalizations in adults, 20112012 influenza season. Clin Infect Disease 2013; $56: 1774-7$

5. Ottenberg AL, Wu JT, Poland GA, et al. Vaccinating health care workers against influenza: the ethical and legal rationale for a mandate. Am J Public Health 2011;101:212-6.

\section{CMAJ 2013. DOI:10.1503/cmaj.113-2123}

We read with interest the CMAJ commentary by Gardam and Lemieux. ${ }^{1}$ Although we agree that a better influenza vaccine is needed, we disagree that this precludes maximizing the use of the current vaccine.
As the authors noted, a recent metaanalysis found vaccination of health care workers was associated with significant reductions in nonspecific adverse patient outcomes. ${ }^{2}$ They also noted that determining which deaths are attributable to influenza is difficult. Given this, nonspecific measures such as influenza-like illness and all-cause mortality are valid measures that may be less likely to miss indirect associations between influenza and mortality.

Vaccine efficacy is higher in healthy health care workers than in frail patients. A 55\%-70\% efficacy rate is far better than the $0 \%$ efficacy of not being vaccinated. Additionally, quoted efficacy rates are often misinterpreted. If 100 health care workers are vaccinated, 55 to 70 of them will be protected. With only half vaccinated, 27 to 35 will be protected, which leaves 65 to 73 vulnerable to infection and potentially spreading influenza to their patients. Which health care worker would you prefer provide care to your loved one?

In 2008, we at BJC HealthCare, in St. Louis, Missouri, started requiring annual influenza vaccination as a condition of employment. ${ }^{3}$ The decision was based on evidence that vaccination of health care workers could protect patients, and that despite substantial efforts made over more than a decade, more than a quarter of our health care workers were not vaccinated. ${ }^{4}$ For the past 5 years, our vaccination rates have remained at about $98 \%$.

We are confident that in the future there will be better efficacy of cleaning products for Clostridium difficile, better gloves to protect patients and health care workers during surgery, and more rapidly effective antibiotics to prevent postoperative infections. While anticipating those advancements, we should not stop thoroughly cleaning patients' rooms, using gloves during surgical procedures, and administering perioperative antibiotics in a timely manner.

While waiting for better tools, we should use all tools currently available to protect our patients now. 